\title{
\begin{tabular}{l|l} 
MitTraries & DSpace@MIT
\end{tabular}
}

\author{
MIT Open Access Articles
}

\section{Model Uncertainty: A Challenge in Nonlinear Coupled Multidisciplinary System Design}

The MIT Faculty has made this article openly available. Please share how this access benefits you. Your story matters.

Citation: Feldstein, Alexander W., et al. "Model Uncertainty: A Challenge in Nonlinear Coupled Multidisciplinary System Design." 2018 AIAA/ASCE/AHS/ASC Structures, Structural Dynamics, and Materials Conference, 8-12 January, 2018, Kissimmee, Florida, American Institute of Aeronautics and Astronautics, 2018.

As Published: https://doi.org/10.2514/6.2018-0652

Publisher: American Institute of Aeronautics and Astronautics

Persistent URL: http://hdl.handle.net/1721.1/116124

Version: Author's final manuscript: final author's manuscript post peer review, without publisher's formatting or copy editing

Terms of use: Creative Commons Attribution-Noncommercial-Share Alike 


\title{
Model Uncertainty: A Challenge in Nonlinear Coupled Multidisciplinary System Design
}

\author{
Alex Feldstein* \\ Massachusetts Institute of Technology, Cambridge, MA, 02139, USA \\ David Lazzara ${ }^{\dagger}$ and Norman Princen ${ }^{\ddagger}$ \\ The Boeing Company, Huntington Beach, CA, 92647, USA \\ Karen Willcox ${ }^{\S}$ \\ Massachusetts Institute of Technology, Cambridge, MA, 02139, USA
}

\begin{abstract}
This paper considers the effects of uncertainty on multidisciplinary analysis estimates used in conceptual aircraft design. Specifically, the paper presents an analysis of the stability and control (S\&C) characteristics of an illustrative Blended-Wing-Body (BWB) configuration using uncertain aerodynamic estimates. The S\&C properties of a BWB are highly nonlinear, and as such pose a challenge for low-fidelity analysis techniques. By propagating bounds on uncertain aerodynamic data them through the analysis, bounds are derived for the resulting S\&C limits. These estimates can then be used to guide future research and development efforts. Results demonstrate that even moderate uncertainty in estimating aerodynamic lift, drag, and moment coefficients translates into large uncertainty in estimating the center of gravity limits, due to the highly nonlinear nature of the S\&C model. The paper identifies directions for ongoing work using a multifidelity approach to address this issue.
\end{abstract}

\section{Introduction}

Quantifying and controlling uncertainty in a conceptual design process is critical for ensuring that the resulting design choices are appropriate. A design under uncertainty process, such as that shown in Figure 1, seeks to quantify the effect of the various sources of uncertainty on the performance of a system. In addition to the traditional design loop closure of the requirements and the performance, additional closure loops are added to meet tolerable levels of uncertainty and cost. Sensitivities of the performance metrics to the uncertain model parameters can then be used to determine where research time and cost should be spent to reduce the input uncertainties. The distributions of the performance metrics can be used to estimate the risk of not meeting the performance requirements. The design is not closed until the risk is reduced to an acceptable level. The relationships between the uncertain distributions assigned to the input parameters and the uncertain distributions induced in the performance metrics and constraints, shown notionally in the forward path of Figure 1, can be complicated and in general must be estimated by numerical simulation (e.g., via stochastic collocation or Monte Carlo simulation). This paper considers the specific case of quantifying how uncertainty in aerodynamic estimates affects estimates of the stability and control (S\&C) characteristics of a Blended-Wing-Body (BWB) aircraft.

Uncertainty can come from many sources, such as from the calculation of regression fit parameters of surrogate models, the calibration of low-fidelity models, and the extrapolation of historical data. Add these modeling uncertainties to those due to attempting to predict future materials, uncertain performance requirements, and uncertain regulations, and the design space is quickly dominated by uncertainty. If the

\footnotetext{
*Graduate Student, Department of Aeronautics and Astronautics, alexfeld@mit.edu, Student Member AIAA

${ }^{\dagger}$ Aerodynamics Engineer, Boeing Research and Technology, Member AIAA

${ }^{\ddagger}$ Blended Wing Body Chief Engineer, Senior Member AIAA

§Professor, Department of Aeronautics and Astronautics, kwillcox@mit.edu, Associate Fellow AIAA
} 


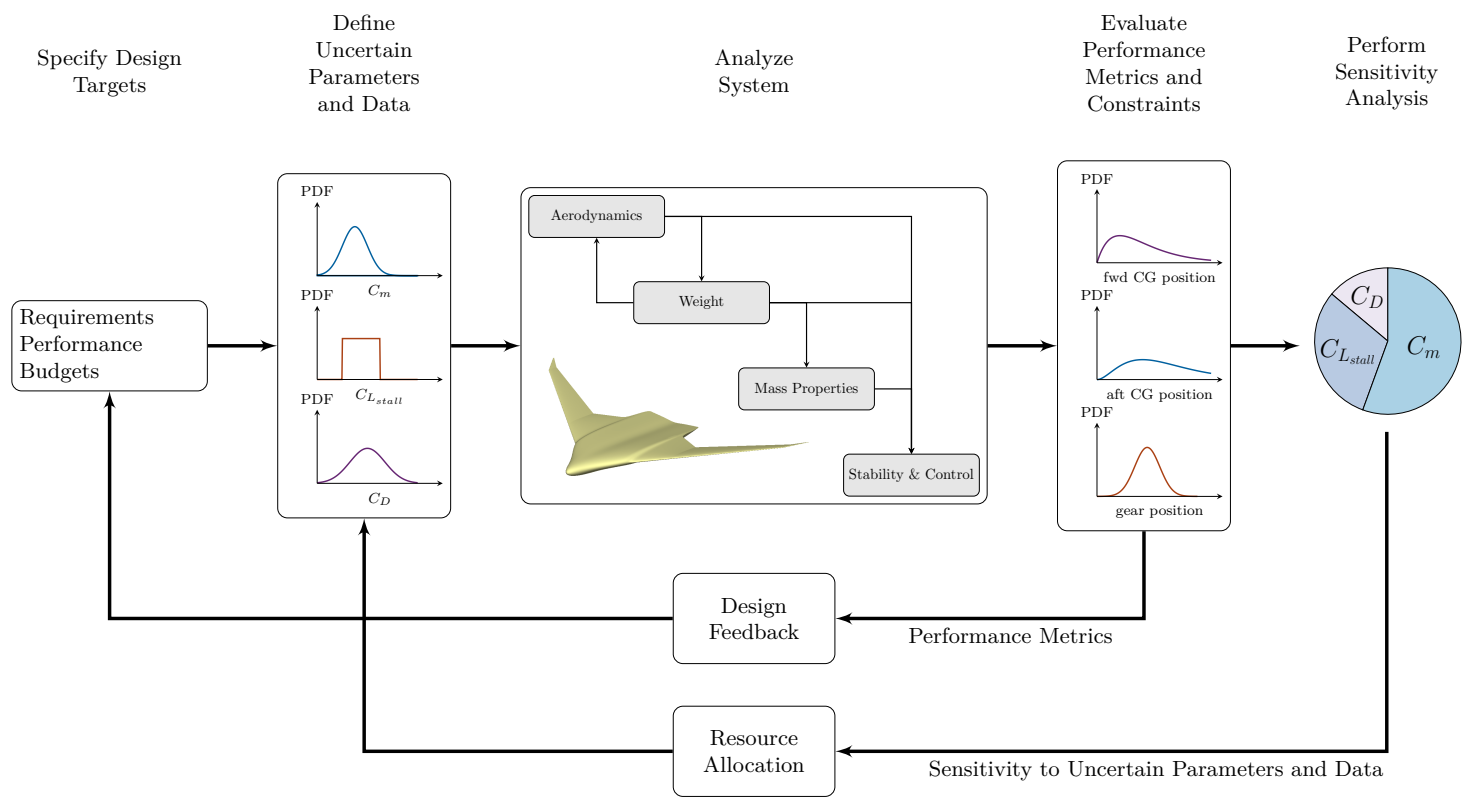

Figure 1: Complex system design under uncertainty with example uncertain parameters and performance metrics. Adapted from Ref. [1].

uncertainties are not adequately quantified, erroneous design decisions made early in the conceptual phase can propagate through the design and production, resulting in increased cost at late stages of a development program.

Prior works analyzed new transport aircraft with uncertain design parameters and have demonstrated the benefits of adding a rigorous consideration of uncertainty to the design and analysis processes. ${ }^{1-4}$ Other works have focused on the propagation of uncertainty and the different types of uncertainties that are present in coupled engineering system design. ${ }^{5-11}$ These works show that consideration of uncertainty expands the design space and often enriches it, allowing for more robust designs to be obtained.

The Blended-Wing-Body (BWB) configuration is designed to serve the long-haul passenger and cargo transport markets. ${ }^{12}$ A key design driver of these configurations is the stability and control (S\&C) analysis, which assesses control effectiveness and computes the operating center of gravity limits. The S\&C analysis relies on accurate estimates of the aircraft's lift, drag, and pitching moment characteristics. ${ }^{13}$ While the low-fidelity models typically used for conceptual design can adequately estimate lift for a BWB over a large portion of the design space, they usually predict a linear relationship between operating parameters, drag, the pitching moment. This differs from the nonlinear dependence usually seen in BWB configurations. Due to the tight coupling among aerodynamics, planform layout, and S\&C in a BWB configuration, it is essential that the S\&C constraints be considered early in the conceptual design process while also developing some understanding of the impact that low-fidelity model uncertainty has on the results.

In this work we present an analysis of the BWB S\&C center of gravity limits using low-fidelity aerodynamic models. We develop uncertainty bounds for the estimated aerodynamic data and other parameters of the analysis. The uncertain data is then run through the S\&C analysis to derive bounds on the performance metrics of interest, namely the fore and aft center of gravity limits, using surrogate models based on Gaussian Process regression. Section II of this paper briefly describes Gaussian Process regression and the combination of surrogate and model uncertainties. Section III presents the disciplinary models relevant to the BWB S\&C analysis. Section IV presents the results, and finally Section V concludes the paper.

\section{Modeling for Analysis Under Uncertainty}

Section II.A presents an overview of Gaussian Process regression, which is used to build surrogate models of the analysis disciplines and provides a platform for synthesizing estimates from multiple fidelities. Section II.B presents the model for representing and combining uncertainties. 


\section{II.A. Gaussian Process Regression}

Gaussian Processes have gained prominence in the surrogate modeling and machine learning communities due to their ability to model complex, multidimensional surfaces while simultaneously providing an explicit measure of confidence in their predictive outputs. ${ }^{14,15}$ Given an input space $\mathcal{D} \in \mathbb{R}^{d}$ and an input vector $\mathbf{x} \in \mathcal{D}$

$$
\mathbf{x}=\left[\begin{array}{c}
x_{1} \\
x_{2} \\
\vdots \\
x_{d}
\end{array}\right]
$$

we wish to create a surrogate model of the output function $f(\mathbf{x})$. In our setting, this function $f$ represents quantities of interest that relate to design performance metrics and constraints. A Gaussian Process is a function

$$
g(\mathbf{x}) \sim \mathcal{G P}(m(\mathbf{x}), k(\mathbf{x}, \mathbf{x}))
$$

with mean function

$$
m(\mathbf{x}): \mathcal{D} \rightarrow \mathbb{R}
$$

and covariance function

$$
k\left(\mathbf{x}, \mathbf{x}^{\prime}\right): \mathcal{D} \times \mathcal{D} \rightarrow \mathbb{R} .
$$

The mean function describes the trends in the output space while the covariance function kernel $k\left(\mathbf{x}, \mathbf{x}^{\prime}\right)$ encodes the relationship between two points, $\mathbf{x}$ and $\mathbf{x}^{\prime}$, in the input space $\mathcal{D}$.

The posterior prediction of the Gaussian Process can be explicitly derived. Let $X$ denote a matrix of $N$ training points,

$$
X=\left[\begin{array}{cccc}
\mid & \mid & & \mid \\
\mathbf{x}_{1} & \mathbf{x}_{2} & \cdots & \mathbf{x}_{N} \\
\mid & \mid & & \mid
\end{array}\right],
$$

where each $\mathbf{x}_{i} \in \mathcal{D}$. Let $\mathbf{f}=\left[f_{1}, f_{2}, \ldots, f_{N}\right]^{T}$ be the column vector containing the corresponding values of the quantity of interest computed by the function being approximated (that is, $f_{i}$ is $f\left(\mathbf{x}_{i}\right)$, the quantity of interest evaluated at the point $\mathbf{x}_{i}$ ). The Gaussian Process prediction at a test point $\mathbf{x}^{*} \in \mathcal{D}$ is given by

$$
\begin{aligned}
& \mu_{G P}\left(\mathbf{x}^{*}\right)=m\left(\mathbf{x}^{*}\right)+k\left(\mathbf{x}^{*}, X\right) k(X, X)^{-1} \mathbf{f} \\
& \sigma_{G P}\left(\mathbf{x}^{*}\right)=k\left(\mathbf{x}^{*}, \mathbf{x}^{*}\right)-k\left(\mathbf{x}^{*}, X\right) k(X, X)^{-1} k\left(X, \mathbf{x}^{*}\right) .
\end{aligned}
$$

where $\mu_{G P}(\mathbf{x})$ is the posterior mean and $\sigma_{G P}(\mathbf{x})$ is the posterior standard deviation. Note that $k\left(X, \mathbf{x}^{*}\right)$ is a column vector where each entry corresponds to the kernel evaluated with $\mathbf{x}^{*}$ and $\mathbf{x}_{i}$ :

$$
k\left(X, \mathbf{x}^{*}\right)=\left[\begin{array}{c}
k\left(\mathbf{x}_{1}, \mathbf{x}^{*}\right) \\
k\left(\mathbf{x}_{2}, \mathbf{x}^{*}\right) \\
\vdots \\
k\left(\mathbf{x}_{N}, \mathbf{x}^{*}\right)
\end{array}\right] .
$$

Similarly, $k\left(\mathbf{x}^{*}, X\right)$ is a row vector, equal to $k\left(X, \mathbf{x}^{*}\right)^{T}$, and $k(X, X)$ is a square, symmetric matrix:

$$
k(X, X)=\left[\begin{array}{cccc}
k\left(\mathbf{x}_{1}, \mathbf{x}_{1}\right) & k\left(\mathbf{x}_{1}, \mathbf{x}_{2}\right) & \cdots & k\left(\mathbf{x}_{1}, \mathbf{x}_{N}\right) \\
k\left(\mathbf{x}_{2}, \mathbf{x}_{1}\right) & \ddots & & \vdots \\
\vdots & & \ddots & \vdots \\
k\left(\mathbf{x}_{N}, \mathbf{x}_{1}\right) & \cdots & \cdots & k\left(\mathbf{x}_{N}, \mathbf{x}_{N}\right)
\end{array}\right] .
$$

The regression process begins by selecting a prior distribution. The prior encodes our a priori belief in the form of the function being approximated. For Gaussian Process regression this consists of choosing a prior mean and covariance function. In this work a zero mean function is used,

$$
m(\mathbf{x})=0 .
$$

3 of 15 
If something is known about the structure of the function being approximated, then the prior mean should be modified accordingly.

For the present work, the square exponential with automatic relevance determination is utilized for the covariance function. The square exponential kernel computes the relationship between two points $\mathbf{x}$ and $\mathbf{x}^{\prime}$ in the input space along each of its dimensions,

$$
k\left(\mathbf{x}, \mathbf{x}^{\prime}\right)=\sigma^{2} \exp \left(-\sum_{i=1}^{d} \frac{\left\|x_{i}-x_{i}^{\prime}\right\|_{2}^{2}}{2 \ell_{i}^{2}}\right) .
$$

This choice of covariance function allows for each dimension [ $]_{i}$ of the $d$-dimensional input space to be weighed relative to the others via the correlation length $\ell_{i}$. This can be useful if one of the inputs has a lower or higher impact on the output relative to the others or if the magnitudes of the input dimensions are distinct from one another. The factor $\sigma^{2}$ can be interpreted as the maximum covariance of the function. If the distance between the two vectors $\mathbf{x}$ and $\mathbf{x}^{\prime}$ is zero (i.e., they are the same point, $\mathbf{x}=\mathbf{x}^{\prime}$ ), then the kernel function returns $\sigma^{2}$. The variables $\sigma$ and $\ell_{i}$ are called hyperparameters. All of the hyperparameters are contained in the vector $\boldsymbol{\theta}$ :

$$
\boldsymbol{\theta}=\left[\begin{array}{c}
\sigma \\
\ell_{1} \\
\ell_{2} \\
\vdots \\
\ell_{d}
\end{array}\right]
$$

The final step of the regression is to choose the hyperparameters. In order to determine the values of the hyperparameters, we maximize the log marginal likelihood, which is conditioned on the training set $X$ :

$$
\ln p(\mathbf{f} \mid X)=-\frac{1}{2} \mathbf{f}^{T} k(X, X)^{-1} \mathbf{f}-\frac{1}{2} \ln |k(X, X)|-\frac{n}{2} \ln 2 \pi .
$$

This leads to the regression problem:

$$
\boldsymbol{\theta}^{*}=\underset{\boldsymbol{\theta}}{\operatorname{argmax}} \ln p(\mathbf{f} \mid X) .
$$

The optimization searches for the choice of $\boldsymbol{\theta}$ that maximizes the probability that the data $\mathbf{f}$ came from the model defined by the hyperparameters $\boldsymbol{\theta}$, conditioned on the training points $X$. Bounds are often added to the hyperparameters to ensure that physically realistic surrogates are constructed.

\section{II.B. Modeling Uncertainty}

Let a given quantity of interest $f$ be modeled by an approximate function $\tilde{f}(\mathbf{x})$ :

$$
\tilde{f}(\mathbf{x}): \mathbb{R}^{d} \rightarrow \mathbb{R}
$$

The function $\tilde{f}(\mathbf{x})$ represents the disciplinary model used to estimate a given quantity of interest. For example, for estimating lift, the function $\tilde{f}(\mathbf{x})$ might represent a low-fidelity vortex lattice model or a higherfidelity computational fluid dynamic model. We distinguish between $f$ and $\tilde{f}$ because we will explicitly account for the uncertainty associated with our imperfect engineering models. We consider a separate model for each output of a given discipline. Thus, in this work each quantity of interest is treated as a scalar.

In order to account for our confidence in the model $\tilde{f}$ we define a fidelity function:

$$
\sigma_{\tilde{f}}(\mathbf{x}): \mathbb{R}^{d} \rightarrow \mathbb{R}
$$

The fidelity function quantifies a standard deviation of the predictions of model from the true value of $f(\mathbf{x})$. Note that the probability density function from which $\sigma_{\tilde{f}}(\mathbf{x})$ is computed is usually unknown and in practice is either assumed or inferred.

For each quantity $\tilde{f}(\mathbf{x})$, we construct a Gaussian Process regression model $g(\mathbf{x})$. The output of the Gaussian Process surrogate is a prediction $\mu_{G P}(\mathbf{x})$ of the model $\tilde{f}(\mathbf{x})$. This prediction has an associated standard deviation $\sigma_{G P}(\mathbf{x})$, which we refer to as the Gaussian Process standard deviation.

In our representation we thus have two sources of uncertainty: the uncertainty associated with the fidelity of the model, quantified by $\sigma_{\tilde{f}}(\mathbf{x})$, and the uncertainty arising from the surrogate modeling process, quantified 
by $\sigma_{G P}(\mathbf{x})$. The total standard deviation of the estimate of $f(\mathbf{x})$, is denoted by $\sigma_{t}(\mathbf{x})$. The corresponding total variance is computed by summing the fidelity and Gaussian Process variances:

$$
\sigma_{t}^{2}(\mathbf{x})=\sigma_{G P}^{2}(\mathbf{x})+\sigma_{\tilde{f}}^{2}(\mathbf{x})
$$

Note that the Gaussian Process standard deviation is zero at the training points. However, the total variance of the estimate of $\tilde{f}(\mathbf{x})$ cannot be lower than the fidelity variance. If the surrogate is perfectly trained (i.e., the Gaussian Process standard deviation is zero), the total standard deviation will be equal to the fidelity standard deviation. This prevents a given surrogate from being treated as too reliable simply because it is better trained. Likewise, including the Gaussian Process standard deviation in the total standard deviation prevents badly trained surrogates, or points far outside of the training set, from being treated as too reliable.

\section{II.C. Uncertainty Propagation}

For this study we only consider the impact of the bounds of the uncertain parameters on the performance metrics. Once the distributions on the input variables have been determined, the minimum and maximum values of the inputs are run through the model to determine the combined impact of the best and worst case inputs. The outputs of this analysis are then the values of the quantity of interest under the best and worst case scenarios.

\section{Blended Wing Body Example Problem}

Unlike conventional aircraft configurations, the planform of the BWB is primarily driven by the arrangement of the passenger and cargo compartments and the stability and control (S\&C) characteristics of the vehicle. ${ }^{13,16}$ The S\&C criteria are highly non-linear due to the three-dimensional nature of the aerodynamic flow and the fact that most of the S\&C criteria involve stall. Many aerodynamic analysis methods are insufficient to model such flow behaviors, often due to their linear formulation or missing physics. This necessitates the use of more expensive high-fidelity analysis to better evaluate S\&C constraints of a BWB configuration. This section presents an overview of the geometry, aerodynamic, propulsion, and S\&C models used to analyze an illustrative BWB example configuration. These models are representative of an academic analysis on a baseline design derived from publicly-available data, and do not necessarily reflect current Boeing BWB design or analysis techniques.

\section{A Note on Axis Systems}

Several reference frames are used in general aircraft analysis for different engineering disciplines. The aerodynamic forces and moments are computed in the stability axes, also called the aerodynamic axes, denoted $(x, y, z)_{\text {aero. }}$. The aerodynamic axes are aligned with the free-stream velocity vector $\vec{V}_{\infty}$. The S\&C analysis in this work is done in the body axes $(x, y, z)_{b o d y}$, which are aligned with the mean chord line of the vehicle. The angle between the body frame $x_{\text {body }}$ and the aerodynamic frame $x_{\text {aero }}$ is the angle of attack, denoted by $\alpha$. The angle between the ground frame $x_{\text {ground }}$ and $x_{\text {body }}$ is the inclination angle $\theta$. The angle between the freestream velocity and the chord line is denoted by $\gamma$. Additionally, all three angles are related:

$$
\theta=\alpha+\gamma
$$

Figure 2 shows these angles and the three axis systems.

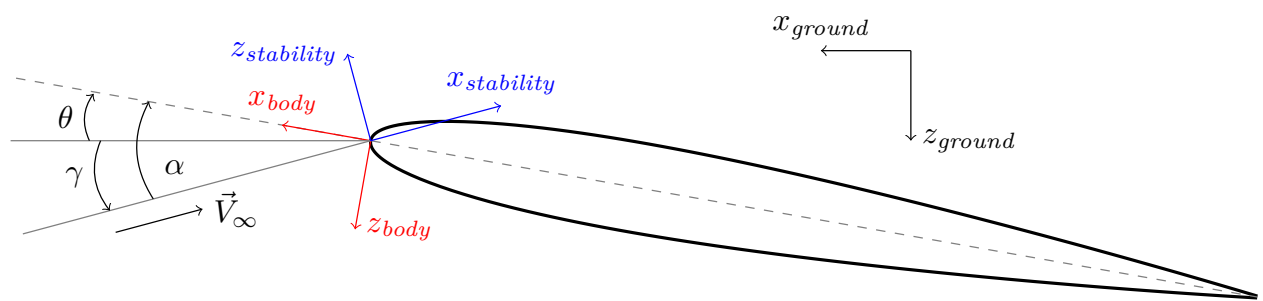

Figure 2: Axis systems used for aircraft analysis. All three systems are right-handed and have the $y$ axis in common. 


\section{III.A. Geometry}

In this example problem, the geometry engine providing configuration information for analysis is Engineering SketchPad (ESP). ${ }^{17}$ ESP links computer aided design (CAD) geometry representations with scripts for generating analysis geometry input files for a variety of programs. The geometry is defined via a set of primitives which are either blended using a cubic spline or ruled using linear interpolation to form surfaces. In this case a BWB configuration is modeled with NACA airfoil primitives.

The BWB analyzed in this paper is inspired by the ERA-0009H1 planform. ${ }^{18}$ The centerbody airfoils are slightly reflexed (inverted NACA 4-series) while the wing airfoils are all symmetric NACA 4-series airfoils. For simplicity, only the wing and centerbody are modeled for the purposes of computing the aerodynamics. A surface rendering of the BWB is shown in Figure 3.

The planform, shown in Figure 4, consists of four elements: a centerbody section, a fairing between the centerbody and panel one of the wing, a two-panel wing, and a winglet. There are thirteen control surfaces cut into the centerbody and wing. The inner three are called the inboard (IB) control surfaces. The control surfaces on the wing are the outboard $(\mathrm{OB})$ control surfaces.

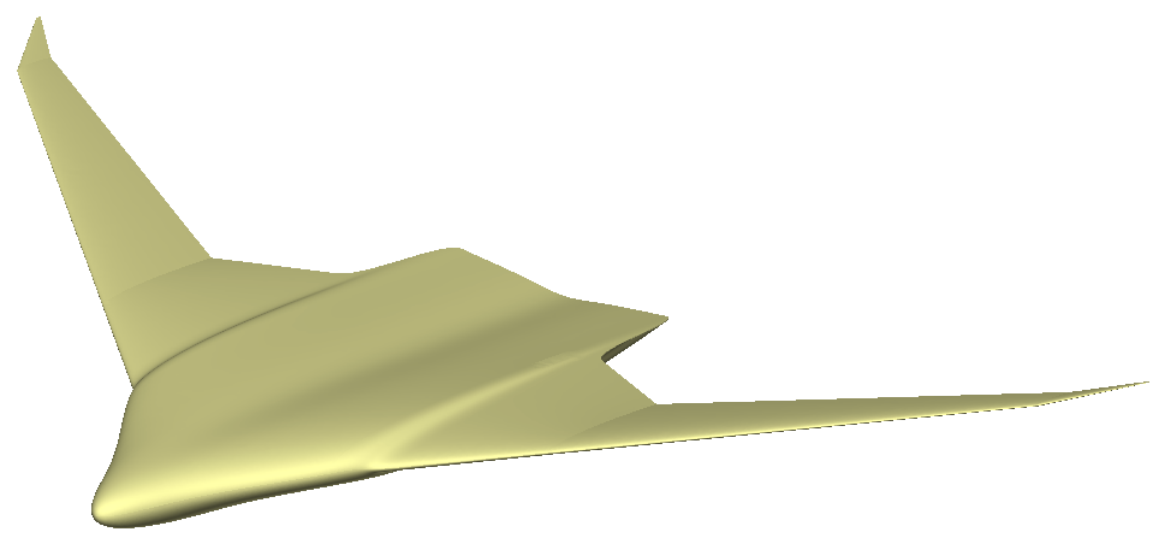

Figure 3: ESP representation of the BWB geometry.

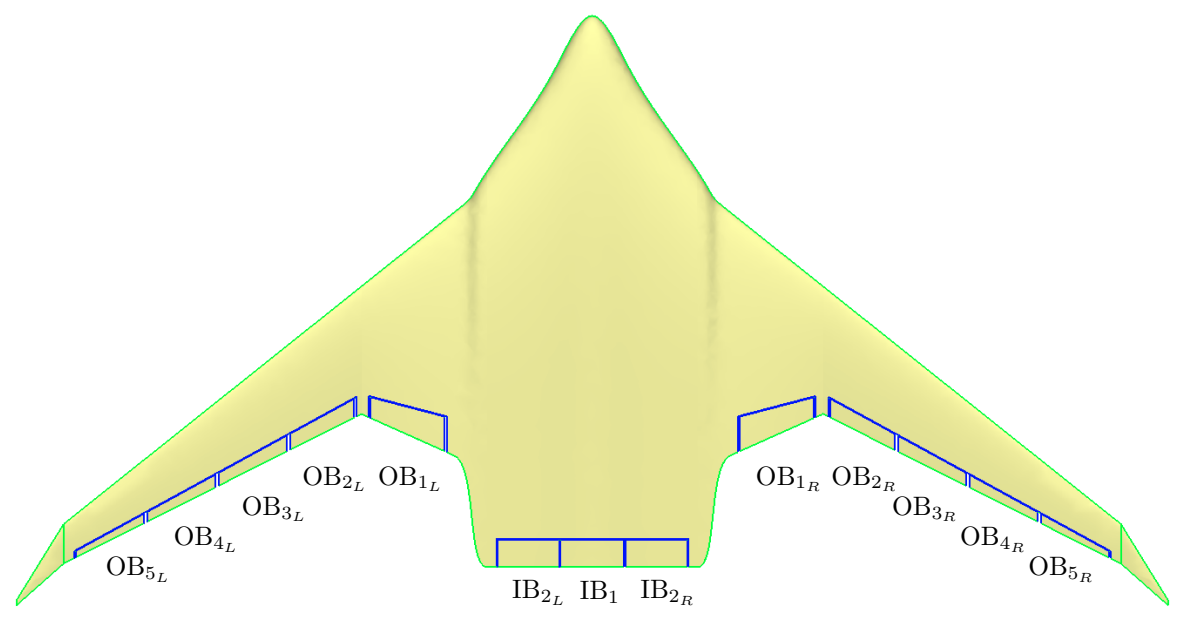

Figure 4: Top view of the BWB planform with the inboard (IB) and outboard (OB) control surfaces denoted.

\section{III.B. Aerodynamics}

In this paper a vortex lattice model is used to evaluate the aerodynamic performance of the BWB configuration. It computes the lift coefficient, $C_{L}$, drag coefficient, $C_{D}$, and pitching moment coefficient about the 
reference point, $C_{m_{r e f}}$. The aerodynamic outputs are functions of the Mach number $M$, the angle of attack $\alpha$, and the deflections of 7 control surfaces $\delta_{1}\left(\mathrm{IB}_{1}\right), \delta_{2}\left(\mathrm{IB}_{2}\right), \delta_{3}\left(\mathrm{OB}_{1}\right), \delta_{4}\left(\mathrm{OB}_{2}\right), \delta_{5}\left(\mathrm{OB}_{3}\right), \delta_{6}\left(\mathrm{OB}_{4}\right)$, and $\delta_{7}\left(\mathrm{OB}_{5}\right)$. Together these inputs make up the vector $\mathbf{x}_{\text {aero }}$.

$$
\mathbf{x}_{\text {aero }}=\left[\begin{array}{lllllllll}
M & \alpha & \delta_{1} & \delta_{2} & \delta_{3} & \delta_{4} & \delta_{5} & \delta_{6} & \delta_{7}
\end{array}\right]^{T}
$$

In this example problem, only longitudinal $\mathrm{S} \& \mathrm{C}$ characteristics are analyzed. Therefore, the left and right control surfaces are combined. Thus, a deflection of $\delta_{1}$ is equivalent to deflecting both $O B_{1_{L}}$ and $O B_{1_{R}}$ in Figure 4. After this simplification, the analysis has $n_{\delta}=7$ control surfaces.

\section{Vortex Lattice Aerodynamic Model}

The vortex lattice model represents aerodynamic surfaces as a collection of bound vortices. The strengths of the vortices are determined by solving a linear system of equations. As a result, the solutions produced by vortex lattice methods are linear. Vortex lattice is useful for determining the lift distribution and induced drag of a configuration. It is an inviscid method, which necessitates the addition of drag corrections to account for viscous effects.

The vortex lattice code used for the present work is Athena Vortex Lattice (AVL). ${ }^{19}$ Although vortex lattice codes do not in general model compressibility effects, AVL implements a Prandtl-Glauert length scaling factor to approximate compressibility effects. The AVL representation of the BWB is shown in Figure 5a. An example solution is shown in Figure 5b. To account for the missing compressibility and viscous effects, a drag coefficient offset is assumed (or calculated through other methods).

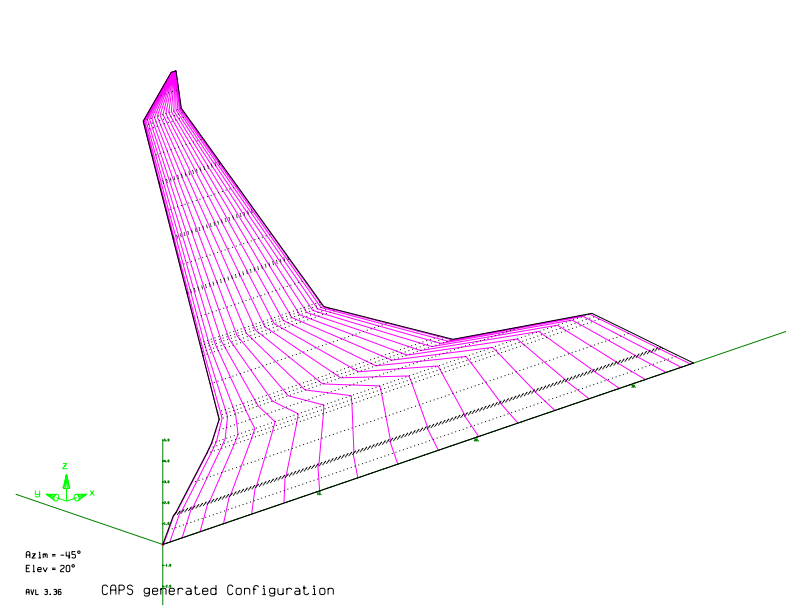

(a) Bound vortex representation of the BWB in AVL.

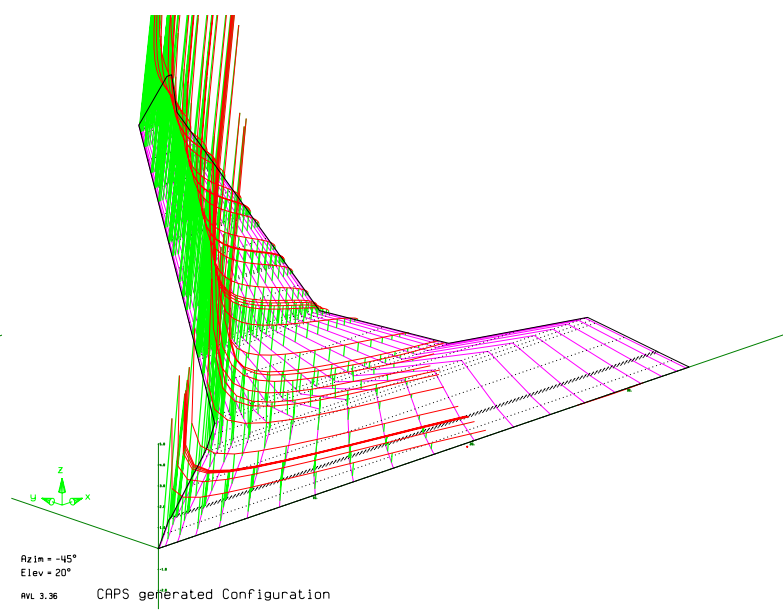

(b) Spanwise delta coefficient of pressure slices.

Figure 5: AVL half planform and solution.

\section{Aerodynamic Fidelity Functions}

As no truth model is available for the modeled aerodynamic quantities, the fidelity functions, denoted as $\sigma_{\left.f_{[]}\right]}$, reflect expert opinion both about the quality of predictive capability of AVL with respect to a BWB configuration. In this case, the fidelity functions are chosen such that for a baseline operating point the prediction of the model is some percentage of the baseline value of the quantity of interest.

The fidelity functions for each quantity compute the baseline uncertainty, i.e., the minimum fidelity uncertainty, as a percentage of the value of the quantity at the baseline point. These percentages are given in Table 1. Each percentage is equated to the $\pm 3 \sigma_{f}$ confidence bound. These confidence bounds have not been proven, but are used here for illustrative purposes. 


\begin{tabular}{cc}
\hline Quantity & Confidence Bound Percentage of Baseline Value \\
\hline$C_{L}$ & $\pm 5 \%$ \\
$C_{D}$ & $\pm 15 \%$ \\
$C_{m_{r e f}}$ & $\pm 10 \%$ \\
\hline
\end{tabular}

Table 1: Example fidelity confidence bounds on $\pm 3 \sigma_{f}$ for each aerodynamic coefficient assigned to AVL at the baseline point.

Due to the low-fidelity nature of AVL, we expect that the error between the model and the true values of the aerodynamic coefficients will grow away from the baseline point. We model the uncertainty as growing linearly with the magnitude of the quantity of interest away from the baseline while keeping the same level of confidence in the prediction. This choice models the decreasing validity of the underlying linear aerodynamics away from the baseline point. This results in the following fidelity functions:

$$
\begin{aligned}
& \sigma_{f_{C_{L}}}\left(\mathbf{x}_{\text {aero }}\right)=\frac{0.05\left(\left|C_{L}\left(\mathbf{x}_{\text {aero }}\right)-C_{L_{\text {base }}}\right|+C_{L_{\text {base }}}\right)}{3} \\
& \sigma_{f_{C_{D}}}\left(\mathbf{x}_{\text {aero }}\right)=\frac{0.15\left(\left|C_{D}\left(\mathbf{x}_{\text {aero }}\right)-C_{D_{\text {base }}}\right|+C_{D_{\text {base }}}\right)}{3}
\end{aligned}
$$

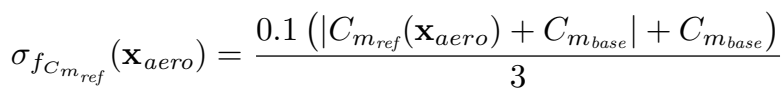

Here $C_{[]_{\text {base }}}$ are the baseline values of $C_{L}, C_{D}$ and $C_{m_{\text {ref }}}$ which are used as the centers of the fidelity functions. The $1 / 3$ factor accounts for the size of the confidence interval being 3 times the fidelity standard deviation on either side of the mean. Note that these fidelity functions are constructed for the purpose of the present example problem only, and other choices are possible. While these functions could provide a basis for further uncertainty quantification activities, they should be rigorously refined for future applications.

\section{Aerodynamic Force Calculation}

The aerodynamic coefficients are computed in the stability reference frame. The side force, yawing moment, and rolling moment are ignored since all of the S\&C cases involve only longitudinal motion. The forces and moments are converted into the body frame using the following equations:

$$
\begin{aligned}
F_{X_{\text {aero }}} & =\left(C_{L} \sin \alpha-C_{D} \cos \alpha\right) S_{r e f} \bar{q} \\
F_{Z_{\text {aero }}} & =-\left(C_{L} \cos \alpha+C_{D} \sin \alpha\right) S_{r e f} \bar{q} \\
C_{m_{C G}} & =C_{m_{r e f}}+\frac{F_{Z_{\text {aero }}}\left(x_{C G}-x_{r e f}\right)-F_{X_{\text {aero }}}\left(z_{C G}-z_{r e f}\right)}{S_{r e f} c_{r e f} \bar{q}} \\
M_{Y_{\text {aero }}} & =C_{m_{C G}} S_{\text {ref }} c_{r e f} \bar{q}
\end{aligned}
$$

Here $S_{r e f}$ is the planform area, $c_{r e f}$ is the mean aerodynamic chord, $\bar{q}$ is the dynamic pressure, and $(x, y, z)_{\text {ref }}$ is the location at which the pitching moment $C_{m_{\text {ref }}}$ was computed by the aerodynamic code. The forces in the body frame due to the aerodynamics are denoted by $F_{X_{\text {aero }}}$ and $F_{Z_{\text {aero }}}$ in the $x$ and $z$ directions, respectively. To compute the pitching moment, the moment coefficient about the reference point $C_{m_{\text {ref }}}$ is transferred to the center of gravity $(x, y, z)_{C G}$. The resulting pitching moment coefficient about the center of gravity is denoted by $C_{m_{C G}}$. This coefficient is then used to compute the moment about the center of gravity due to the aerodynamic forces $M_{Y_{\text {aero }}}$.

\section{III.C. Propulsion}

The propulsion model utilized in this work is taken from TASOPT. ${ }^{20}$ It is a thermodynamic model of a gas turbofan engine. The model first sizes the engine based on thrust and combustor stagnation temperature requirements at the beginning of cruise. For this work it is assumed that cruise starts at the maximum takeoff weight, at 41000 feet of altitude, and at a Mach number of 0.85 . This is a conservative assumption that ensures the resulting engine has a safety margin of thrust.

The model computes off-design conditions by iterating on the thermodynamic governing equations using a Newton scheme. The propulsion model computes the total thrust $F_{N_{t o t}}$ in the engine body frame. To compute 
the force of engine in the aircraft body frame, the total thrust is rotated by the engine installation angles. The engines are assumed to be placed symmetrically about the $x z$ plane to cancel out their contributions to roll or yaw moments, or to side force. This leaves the longitudinal and vertical forces in the aircraft frame, denoted by $F_{X_{\text {thrust }}}$ and $F_{Z_{\text {thrust }}}$, respectively, and the pitching moment due to the thrust $M_{Y_{\text {thrust }} \text {. The total }}$ thrust is summed over the number of engines $n_{\text {eng }}$ :

$$
\begin{aligned}
F_{N_{\text {tot }}} & =\sum_{i=1}^{n_{\text {eng }}} F_{N, i} \\
F_{X_{\text {thrust }}} & =F_{N_{\text {tot }}} \cos \psi \\
F_{Z_{\text {thrust }}} & =-F_{N_{\text {tot }}} \sin \psi \\
M_{Y_{\text {thrust }}} & =F_{Z_{\text {thrust }}}\left(x_{C G}-x_{\text {eng }}\right)-F_{X_{\text {thrust }}}\left(z_{C G}-z_{\text {eng }}\right)
\end{aligned}
$$

Here $\psi$ denotes the installation incidence angle of the engine, which is the angle between the engine centerline and the mean chordline of the aircraft. The thrust vector is defined to originate at $(x, y, z)_{\text {eng }}$.

\section{III.D. Stability and Control}

The goal of the S\&C analysis is to compute the flight center of gravity limits and to characterize the controllability of the aircraft. In this work only the longitudinal stability criteria are analyzed. These criteria compute the forward and aft center of gravity limits for the BWB as well as the location of the main landing gear along the longitudinal axis of the vehicle.

We assume that the aircraft is symmetric about the $x z$ plane, quasi-rigid, and fixed mass. Under these assumptions, and with the additional assumption that the criteria are quasi-steady, the equations of motion in the body axes simplify to the following:

$$
\begin{aligned}
m(\dot{U}+g \sin \theta) & =F_{X_{\text {aero }}}+F_{X_{\text {thrust }}}+F_{X_{\text {gear }}} \\
m(\dot{W}-g \cos \theta) & =F_{Z_{\text {aero }}}+F_{Z_{\text {thrust }}}+F_{Z_{\text {gear }}} \\
\dot{Q} I_{y y} & =M_{Y_{\text {aero }}}+M_{Y_{\text {thrust }}}+M_{Y_{\text {gear }}}
\end{aligned}
$$

Here $m$ denotes the mass of the vehicle, $\dot{U}$ is the acceleration along the $x_{b o d y}$ axis, $\dot{W}$ is the acceleration along the $z_{\text {body }}$ axis, $g$ is the acceleration due to gravity, $\theta$ is the angle between the ground and the chord line, $\dot{Q}$ is the rotational acceleration about the $y$ axis, and $I_{y y}$ is the moment of inertia about the $y$ axis. The external forces and moments, $F_{X_{\text {aero }}}, F_{Z_{\text {aero }}}, M_{Y_{\text {aero }}}, F_{X_{\text {thrust }}}, F_{Z_{\text {thrust }}}$, and $M_{Y_{\text {thrust }}}$ are computed using Equations 23, 24, 26, 28, 29, and 30, respectively. The landing gear forces and pitching moment are given by $F_{X_{\text {gear }}}, F_{Z_{\text {gear }}}$, and $M_{Y_{\text {gear }}}$. When the aircraft is in the air the gear forces and moment are set to zero.

For the ground-based criteria we model the landing gear as a nose gear and two main gear. A diagram of the axis systems is shown in Figure 6. The main gear are modeled as symmetric about the $x z$ plane at a distance $\Delta X_{m g}$ along $x_{b o d y}$ and $\Delta Z_{m g}$ along $z_{b o d y}$ from the center of gravity. Similarly, the nose gear is a distance $\Delta X_{n g}$ along the $x_{b o d y}$ axis and $\Delta Z_{n g}$ along the $z_{b o d y}$ axis from the center of gravity.

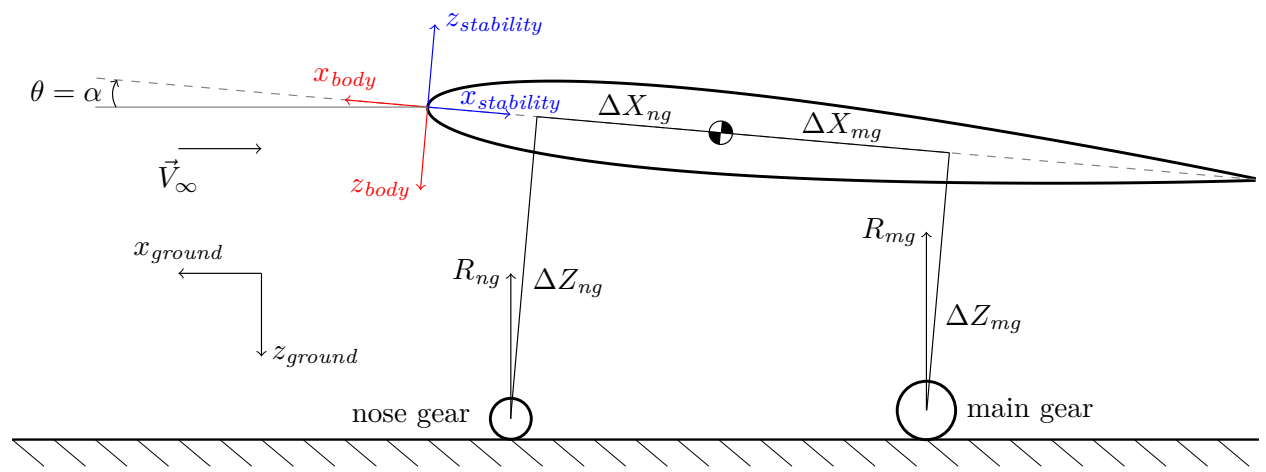

Figure 6: Axis systems for the BWB on the ground along with the gear reaction forces $R_{m g}$ and $R_{n g}$. 
The longitudinal S\&C criteria originate from the United States Federal Aviation Regulations (FAR) Part 25. There are four criteria, two for the forward center of gravity limit, one for the aft limit, and one to set the main gear location along the longitudinal axis of the vehicle. We now briefly describe each criterion.

Fly-To-Stall

The fly-to-stall criterion requires that the control system be able to achieve stall. It is evaluated by trimming the aircraft at 1.13 and 1.3 times the stall speed and then flying it to stall with the control devices. The objective of the criterion is to find the furthest forward center of gravity limit such that the aircraft can be controlled to stall. The speed that produces the furthest aft limit is used as the critical speed.

\section{Stall Recovery}

After the aircraft flies to stall it must be able to recover at a specified rotation rate. The FAR requires "prompt" recovery, which has been interpreted to mean a recovery rotation rate of at least $-5 \mathrm{deg} / \mathrm{s}^{2} .{ }^{21}$ The objective of this criterion is to find the furthest aft center of gravity limit where this rotation requirement is met. The criterion is evaluated at both 1.13 and 1.3 times the stall speed, and with no thrust, idle thrust, and full thrust in order to determine which case is the most critical.

\section{Nose Wheel Steering}

The nose wheel steering criterion sets the longitudinal location of the main gear. Using Ref. [22], the main gear are placed such that at a minimum $6 \%$ of the aircraft's weight is on the nose gear at maximum takeoff weight. For this criterion, the center of gravity is placed at the aft limit as determined by the free flight cases. The computed main gear location is then propagated through the rest of the ground based analyses. This is done to create the widest possible center of gravity travel range.

\section{Nose Wheel Liftoff}

The nose wheel liftoff criterion specifies the pitch-up rotation rate of the aircraft during takeoff. From Ref. [23], a minimum rotation rate of $3 \mathrm{deg} / \mathrm{s}^{2}$ is required when the pitch control surfaces are fully deflected to give an upward rotation. The computed center of gravity is a forward limit and may be further aft than the fly-to-stall limit.

\section{Results}

The BWB example problem includes the disciplines listed above, as well as weight and mass properties analyses that compute the component weights, weight-based centers of gravity, and moments of inertia. These disciplines and their models reflect an academic analysis of the BWB configuration. Gaussian Process surrogates are built for the aerodynamic analysis. The surrogates take in the vector $\mathbf{x}_{\text {aero }}$ (Eq. 19) as input and estimate the output quantities $C_{L}$ (lift coefficient), $C_{D}$ (drag coefficient), and $C_{m_{\text {ref }}}$ (pitching moment coefficient about the reference point). One surrogate model is built for each output quantity of interest. Latin hypercube sampling is used to select 900 sample points for surrogate training. These samples are generated using the input variable bounds listed in Table 2 .

Surrogate models of the AVL estimates for the lift, drag, and pitching moment coefficient without control surface deflections are shown in Figure 7 . The uncertainty bounds in the plots are $\pm 3 \sigma_{t}$. The uncertainty away from the baseline point is dominated by the fidelity standard deviation computed in Equations 20, 21, and 22 .

To visualize the center of gravity design space, the pitching moment about the center of gravity is computed versus the angle of attack for three positions of the center of gravity. These plots are shown in Figure 8. The center of gravity locations are given as percentages of the mean aerodynamic chord MAC. The total effect of the uncertainty in the aerodynamic estimates is clearly seen, noting that the calculation of $C_{m_{C G}}$ about the center of gravity involves all three aerodynamic coefficients, as shown in Equation 25.

Evaluating each S\&C criterion consists of solving an optimization problem to find the furthest forward feasible center of gravity location for the forward limits and the furthest aft location for the aft limits. Feasibility is contingent upon the equations of motion being satisfied as well as any other criterion specific

10 of 15 


\begin{tabular}{ccc}
\hline Input & Bounds & Units \\
\hline Mach & {$[0,0.4]$} & None \\
$\alpha$ & {$[0,25]$} & degrees \\
$\delta_{1}$ & {$[-45,45]$} & degrees \\
$\delta_{2}$ & {$[-45,45]$} & degrees \\
$\delta_{3}$ & {$[-45,45]$} & degrees \\
$\delta_{4}$ & {$[-45,45]$} & degrees \\
$\delta_{5}$ & {$[-45,45]$} & degrees \\
$\delta_{6}$ & {$[-45,45]$} & degrees \\
$\delta_{7}$ & {$[-45,45]$} & degrees \\
\hline
\end{tabular}

Table 2: Input variable bounds for the aerodynamic surrogates.

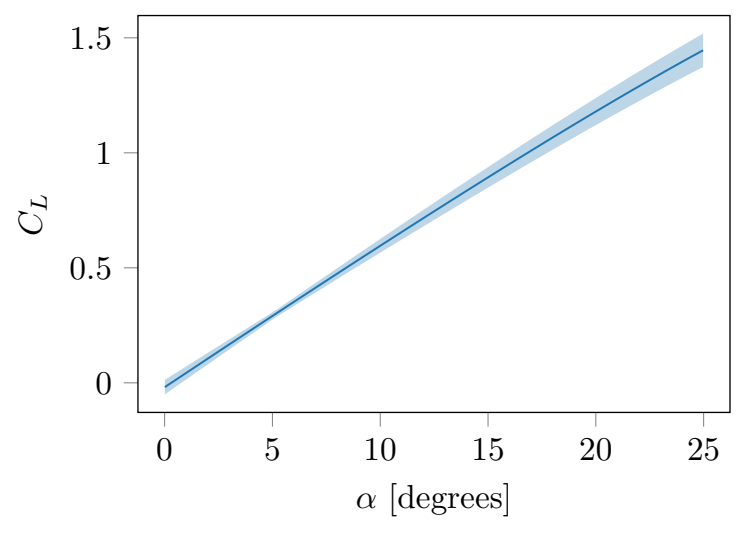

(a) $C_{L}$

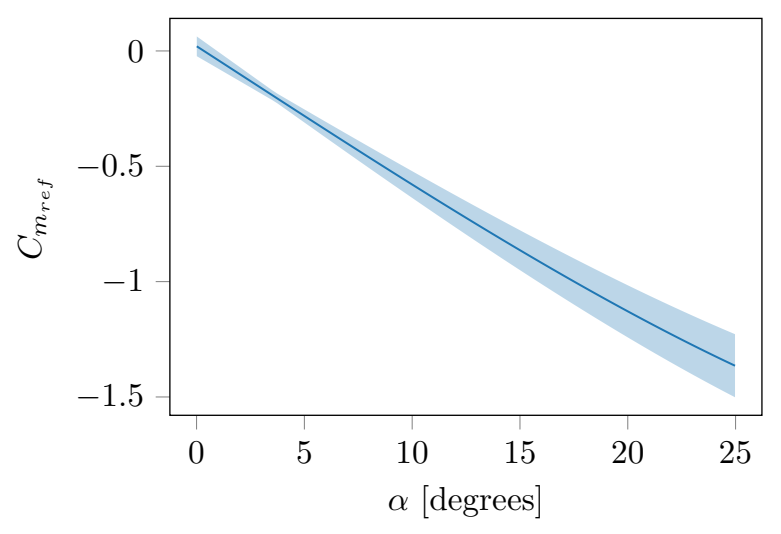

(b) $C_{m_{\text {ref }}}$

Figure 7: AVL aerodynamic coefficient estimates at Mach 0.3 with $\pm 3 \sigma_{t}$ confidence bounds with no control surface deflections.

constraints. This results in a non-linear system of equations. Our studies have shown that these equations often have multiple solutions and can be numerically ill-conditioned. In some cases, we observe high sensitivities to parameter values. These challenges further highlight the difficulty of modeling the $\mathrm{S} \& \mathrm{C}$ characteristics of the BWB.

We first give the deterministic results of the analysis. The $\mathrm{S} \& \mathrm{C}$ criteria are evaluated using control surfaces $\mathrm{IB}_{1}, \mathrm{IB}_{2}, \mathrm{OB}_{1}$, and $\mathrm{OB}_{2}$ as the trim surfaces for the free-flight criteria, and by using all of the surfaces except the two outboard most, $\mathrm{OB}_{4}$ and $\mathrm{OB}_{5}$, for takeoff. The stall lift coefficient was chosen to be $C_{L_{\max }}=1.0$. The resulting center of gravity limits are summarized in Table 3 . The center of gravity

\begin{tabular}{cccc}
\hline Case & Type & CG Location & Gear Location \\
\hline Fly-to-Stall & forward & 62.5 feet & N/A \\
Stall Recovery & aft & 66.6 feet & N/A \\
Nose Wheel Steering & aft & 66.6 feet & 71.0 feet \\
Nose Wheel Liftoff & forward & 62.3 feet & 71.0 feet \\
\hline
\end{tabular}

Table 3: Center of gravity limits computed by each $\mathrm{S} \& \mathrm{C}$ criterion.

travel range computed by the analysis is 4.1 feet. The critical criteria are fly-to-stall and stall recovery. This center of gravity travel range may be sufficient for closing the aircraft, yet it is possible a better one could be derived by changing the sizes of the control surfaces, the stall point, or the control allocation. 


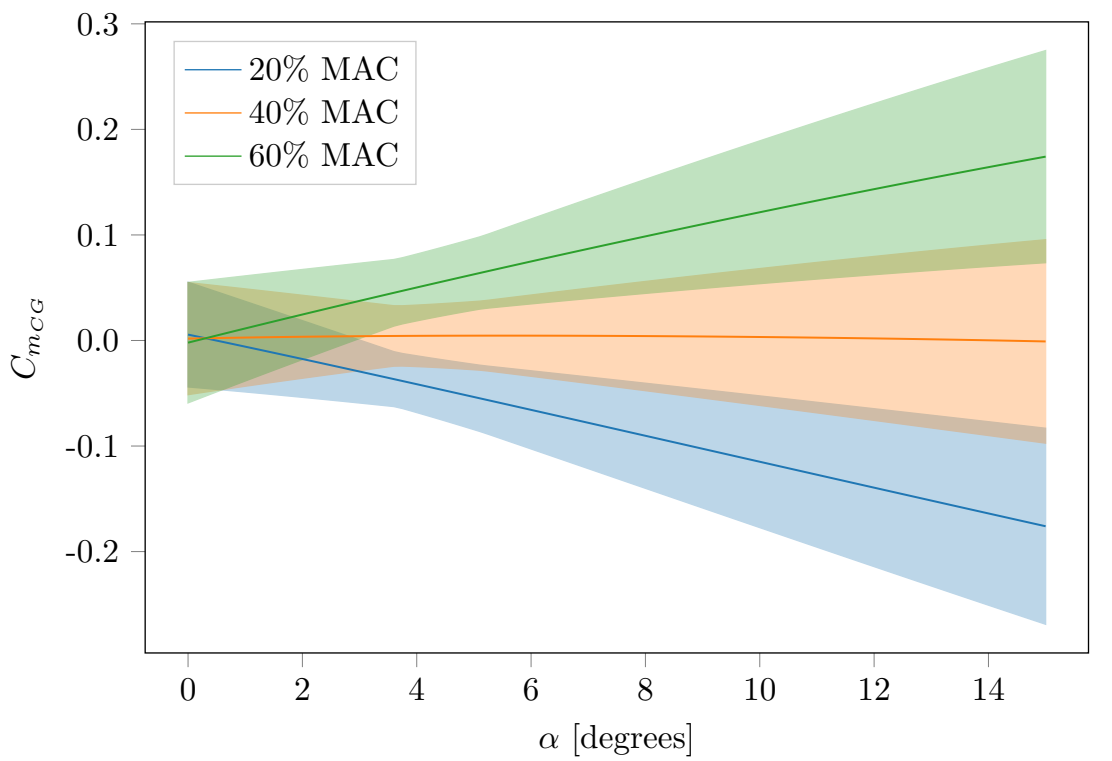

Figure 8: Estimates of the pitching moment about the center of gravity computed using Eq. 25 and the aerodynamic coefficints in Figure 7 . The shaded areas represent the $\pm 3 \sigma_{t}$ confidence bounds.

We next examine the impact of uncertainty in the aerodynamic coefficient predictions on the S\&C center of gravity limits. To do this, best and worst case combinations of the aerodynamic data are considered. Numerical simulations indicate that the largest center of gravity travel range is obtained, in this case, when:

$$
\begin{aligned}
C_{L}^{+} & =C_{L}+n \sigma_{t_{C_{L}}} \\
C_{D}^{+} & =C_{D}-n \sigma_{t_{C_{D}}} \\
C_{m_{\text {ref }}}^{+} & =C_{m_{\text {ref }}}+n \sigma_{t_{C_{m_{r e f}}}}
\end{aligned}
$$

Thus, $C_{L}^{+}, C_{D}^{+}$, and $C_{m_{r e f}}^{+}$represent the positive limits of the lift, drag, and pitching moment coefficients, respectively. Similarly, the negative limits for the lift $C_{L}^{-}$, drag $C_{D}^{-}$, and pitching moment $C_{m_{r e f}}^{-}$coefficients are chosen to narrow the center of gravity travel range:

$$
\begin{aligned}
C_{L}^{-} & =C_{L}-n \sigma_{t_{C_{L}}} \\
C_{D}^{-} & =C_{D}+n \sigma_{t_{C_{D}}} \\
C_{m_{r e f}}^{-} & =C_{m_{r e f}}-n \sigma_{t_{C_{m_{r e f}}}}
\end{aligned}
$$

The parameter $n$ is an input to the model and represents the level of confidence that we have in the aerodynamic data. The lower the value of $n$ the higher the degree of confidence that we have in the estimate of the data.

Table 4 shows the range of changes in the center of gravity limits as a result of uncertainty in the aerodynamic coefficients. To obtain these results, we first set $n=1$ and evaluate the aerodynamic coefficients at their positive limits $\left(C_{L}^{+}, C_{D}^{+}, C_{m_{r e f}}^{+}\right)$and their negative limits $\left(C_{L}^{-}, C_{D}^{-}, C_{m_{r e f}}^{-}\right)$. The $\mathrm{S} \& \mathrm{C}$ analysis is then carried out using each set of aerodynamic coefficients. We repeat the analysis for $n=2$ and $n=3$. For each case in Table 4, the first number represents the center of gravity limit computed using the positive limits for the aerodynamic coefficients, while the second number represents the center of gravity limit computed using the negative limits. Thus, these numbers represent changes in the size of the S\&C uncertainty bounds due to the aerodynamic uncertainty bounds. These data should not be interpreted as confidence intervals, since no probability distributions have been used to define the inputs, nor do they represent hard limits on the uncertainty bounds. Rather, these should be interpreted only as possible changes in the size of the uncertainty bounds as the aerodynamic data become more uncertain. For this analysis, the stall lift coefficient was again set at $C_{L_{\max }}=1.0$ and the same control allocation was used as before. 


\begin{tabular}{ccccc}
\hline Case & Type & $\boldsymbol{n}=\mathbf{1}$ & $\boldsymbol{n = 2}$ & $\boldsymbol{n}=\mathbf{3}$ \\
\hline Fly-To-Stall & forward & {$[59.2,65.8]$} & {$[56.0,69.3]$} & {$[53.0,73.0]$} \\
Stall Recovery & aft & {$[63.3,70.0]$} & {$[60.1,73.5]$} & {$[57.0,77.1]$} \\
Nose Wheel Steering & main gear location & {$[67.4,74.6]$} & {$[64.0,78.4]$} & {$[60.6,82.3]$} \\
Nose Wheel Liftoff & forward & {$[53.2,71.7]$} & {$[44.2,80.7]$} & {$[35.1,89.3]$} \\
\hline CG Travel Range & & {$[4.1,-1.7]$} & {$[4.1,-7.2]$} & {$[4.0,-12.2]$} \\
\hline
\end{tabular}

Table 4: Changes in the center of gravity limit locations corresponding to each S\&C criterion for different levels of uncertainty in the aerodynamic coefficient estimates.

The results in this study show that the uncertainty in the aerodynamic coefficient estimates have a large impact on the predicted center of gravity limits. For each level of uncertainty, there are many instances of a closed design, but taking the worst-case uncertainty levels for the aerodynamic coefficients produces negative center of gravity travel ranges. This indicates that our low-fidelity model introduces enough uncertainty that it is difficult to state with high confidence that the aircraft design is closed, even if the deterministic results are satisfactory. There is still significant uncertainty that needs to be reduced through higher fidelity analysis, since it is known that BWB vehicles have been designed with feasible CG ranges that were successfully demonstrated in flight tests. ${ }^{24}$ This is particularly important for a BWB because the center of gravity travel range is used to set the size of the control surfaces, place the landing gear, and develop strategies for the loading of cargo and fuel. Additionally, work should be done on refining the low-fidelity confidence bounds on the AVL estimates of the aerodynamic coefficients.

It is critical to note that in practice the S\&C analysis is only one part of the larger system analysis. For example, the results in Table 4 show that the Nose Wheel Liftoff case is the most critical using the worst case aerodynamic data and in fact is the only reason the resulting center of gravity travel ranges are negative. In practice the Nose Wheel Liftoff criterion can be relaxed by allowing the aircraft to slightly over-speed on takeoff. This could allow the S\&C analysis to close even though the Nose Wheel Liftoff criterion is not strictly met, as long as the level of uncertainty in the Nose Wheel Liftoff limit is able to be overcome through over-speeding on takeoff. However, allowing the aircraft to over-speed impacts the field length constraint analysis, as over-speeding increases the landing and takeoff field lengths. This in turn impacts braking, weight, and other performance requirements. Thus, a balance must be struck between allowing for some uncertainty in the analysis of Nose Wheel Liftoff and the design of the rest of the system.

This coupling between the different disciplines highlights the need to define uncertainty requirements for each part of the analysis. For example, a low-speed condition where the aircraft is well behaved over a large portion of the operating space may be allowed to carry more margin, i.e., more uncertainty, than a cruise condition, where small perturbations to the aircraft's attitude, speed, or weight can have large impacts on its ability to perform the mission for which it is intended. Therefore, acceptable levels of uncertainty should be assigned to each quantity in each part of the overall system analysis and quantified with respect to any uncertain data or parameters that are present. This brings the uncertainty into the design space where it can be traded against the other design variables, just like any other design parameter such as wing span or cruise Mach number.

\section{Conclusion}

This paper highlights the impact of aerodynamic uncertainty on S\&C estimates for an example BWB vehicle. This is due to the high degree of nonlinearity in the S\&C model. When relying on highly uncertain aerodynamic data from low-fidelity models, the resulting S\&C uncertainty may be sufficiently large to confound conclusions about whether a given vehicle is feasible or infeasible - clearly a level of uncertainty that is unacceptable for making important early stage design decisions.

These results highlight the critical need for bringing higher-fidelity aerodynamic data into the design process to reduce the uncertainty. It is not clear what, if any, level of CFD simulation will be sufficient for accurately capturing the important nonlinear behaviors present in this problem. To do so will require multifidelity and surrogate-based strategies to address computational costs. Multifidelity analysis aims to 
combine the speed of low-fidelity analysis with the confidence of high-fidelity models to efficiently and effectively explore complex, non-linear design spaces. An effective multifidelity methodology requires that the uncertainty associated with each analysis model be quantified, either relative to other model estimates or relative to some truth model (or truth data). This then permits a principled approach to combining estimates from multiple models, while taking into account their relative predictive abilities and relative costs. The Gaussian process surrogate models and uncertainty models used in this paper provide a natural setting in which to introduce multiple fidelity analyses, using the framework of Ref. [25].

\title{
Acknowledgments
}

This work was funded by The Boeing Company.

\section{References}

\begin{abstract}
${ }^{1}$ Opgenoord, M. M. J. and Willcox, K. E., "Sensitivity Analysis Methods for Uncertainty Budgeting in System Design," AIAA Journal, Vol. 54, No. 10, 2016, pp. 3134-3148.

${ }^{2}$ Mavris, D. N., Bandte, O., and DeLaurentis, D. A., "Robust Design Simulation: A Probabilistic Approach to Multidisciplinary Design," Journal of Aircraft, Vol. 36, No. 1, 1999, pp. 298-307.

${ }^{3}$ Barros, P., Kirby, M., and Mavris, D., "A Review of Calibration under Uncertainty within the Environmental Design Space," 47th AIAA Aerospace Sciences Meeting including The New Horizons Forum and Aerospace Exposition, American
\end{abstract} Institute of Aeronautics and Astronautics, Reston, Virigina, 2009.

${ }^{4} \mathrm{Ng}$, L. W.-T. and Willcox, K. E., "A Multifidelity Approach to Aircraft Conceptual Design Under Uncertainty," 10th AIAA Multidisciplinary Design Optimization Conference, American Institute of Aeronautics and Astronautics, Reston, Virginia, 2014, pp. 1-13.

${ }^{5}$ Roy, C. J. and Oberkampf, W. L., "A comprehensive framework for verification, validation, and uncertainty quantification in scientific computing," Computer Methods in Applied Mechanics and Engineering, Vol. 200, No. 25-28, 2011, pp. $2131-2144$.

${ }^{6}$ Aughenbaugh, J. M. and Paredis, C. J. J., "The Value of Using Imprecise Probabilities in Engineering Design," Journal of Mechanical Design, Vol. 128, No. 4, 2006, pp. 969.

${ }^{7}$ Mullur, A., Hajela, P., and Bahei-El-Din, Y., "Uncertainty Management in Design Optimization of Coupled Systems," 11th AIAA/ISSMO Multidisciplinary Analysis and Optimization Conference, American Institute of Aeronautics and Astronautics, Reston, Virigina, 2006.

${ }^{8}$ Seshadri, P., Constantine, P., and Iaccarino, G., "Aggressive Design Under Uncertainty," 16th AIAA Non-Deterministic Approaches Conference, American Institute of Aeronautics and Astronautics, Reston, Virginia, 2014.

${ }^{9}$ Lamorte, N., Friedmann, P. P., Glaz, B., Culler, A. J., Crowell, A. R., and McNamara, J. J., "Uncertainty Propagation in Hypersonic Aerothermoelastic Analysis," Journal of Aircraft, Vol. 51, No. 1, 2014, pp. 192-203.

${ }^{10}$ Ghoreishi, S. F. and Allaire, D. L., "Adaptive Uncertainty Propagation for Coupled Multidisciplinary Systems," AIAA Journal, Vol. 55, No. 11, 2017, pp. 3940-3950.

${ }^{11}$ Lee, S. H. and Chen, W., "A comparative study of uncertainty propagation methods for black-box-type problems," Structural and Multidisciplinary Optimization, Vol. 37, No. 3, 2009, pp. 239-253.

${ }^{12}$ Liebeck, R. H., "Design of the Blended Wing Body Subsonic Transport," Journal of Aircraft, Vol. 41, No. 1, 2004, pp. $10-25$.

${ }^{13}$ Cameron, D. and Princen, N., "Control Allocation Challenges and Requirements for the Blended Wing Body," AIAA Guidance, Navigation, and Control Conference and Exhibit, American Institute of Aeronautics and Astronautics, Reston, Virigina, 2000.

${ }^{14}$ Rasmussen, C. E. and Williams, C. K. I., Gaussian Process for Machine Learning, MIT Press, Cambridge, MA, 2006.

${ }^{15}$ Forrester, A. I., Sobester, A., and Keane, A. J., Engineering Design via Surrogate Modelling: A Practical Guide, American Institute of Aeronautics and Astronautics, Reston, Virginia, 2008.

${ }^{16}$ Liebeck, R., Page, M., and Rawdon, B., "Blended-wing-body subsonic commercial transport," 36th AIAA Aerospace Sciences Meeting and Exhibit, American Institute of Aeronautics and Astronautics, Reston, Virigina, 1998.

${ }^{17}$ Haimes, R. and Dannenhoffer, J., "The Engineering Sketch Pad: A Solid-Modeling, Feature-Based, Web-Enabled System for Building Parametric Geometry," 21st AIAA Computational Fluid Dynamics Conference, American Institute of Aeronautics and Astronautics, Reston, Virginia, 2013.

${ }^{18}$ Quinlan, J. and Gern, F. H., "Conceptual Design and Structural Optimization of NASA Environmentally Responsible Aviation (ERA) Hybrid Wing Body Aircraft," 57th AIAA/ASCE/AHS/ASC Structures, Structural Dynamics, and Materials Conference, American Institute of Aeronautics and Astronautics, Reston, Virginia, 2016, pp. 1-16.

${ }^{19}$ Mark Drela and Harold Youngren, "Athena Vortex Lattice," http://web.mit.edu/drela/Public/web/avl/.

${ }^{20}$ E. M. Greitzer, Bonnefoy, P. A., Blanco, E. D. 1. R., Dorbian, C. S., Drela, M., Hall, D. K., Hansman, R. J., Hileman, J. I., Liebeck, R. H., Lovegren, J., Mody, P., Pertuze, J. A., Sato, S., Spakovszky, Z. S., Tan, C. S., Hollman, J. S., Duda, J. E., Fitzgerald, N., Houghton, J., Kerrebrock, J. L., Kiwada, G. F., Kordonowy, D., Parrish, J. C., Tylko, J., Wen, E. A., and Lord, W. K., "N+3 Aircraft Concept Designs and Trade Studies, Final Report," Tech. rep., National Aeronautics and Space Administration, 2010.

${ }^{21}$ Schuler, J. M. and Dahl, M. A., "Proposed Revisions to MIL-F-8785C Related to Flight Safety of Augmented Aircraft," Tech. rep., United States Air Force Wright Aeronautical Laboratories, 1982. 
${ }^{22}$ Currey, N. S., Aircraft Landing Gear Design: Prinicples and Practices, American Institute of Aeronautics and Astronautics, 1988.

${ }^{23}$ Kroo, I., "Aircraft Design - AA241," https://web.archive.org/web/20170301222042/http://adg.stanford.edu/aa241/ AircraftDesign.html.

${ }^{24}$ Risch, T., Cosentino, G., Regan, C., Kisska, M., and Princen, N., "X-48B Flight Test Progress Overview," 47th AIAA Aerospace Sciences Meeting including The New Horizons Forum and Aerospace Exposition, American Institute of Aeronautics and Astronautics, Reston, Virigina, 2009.

${ }^{25}$ Lam, R., Allaire, D., and Willcox, K., "Multifidelity Optimization using Statistical Surrogate Modeling for NonHierarchical Information Sources," 56th AIAA/ASCE/AHS/ASC Structures, Structural Dynamics, and Materials Conference, 2015 . 